\title{
Psychopharmacological options for adult patients with anorexia nervosa: the patients' and carers' perspectives
}

\author{
Hubertus Himmerich, ${ }^{1,2 *}$ Moradeke Joaquim, ${ }^{2}$ Jessica Bentley, ${ }^{2}$ Carol Kan, \\ Julia Dornik, ${ }^{1,2}$ Janet Treasure, ${ }^{1,2}$ and Ulrike Schmidt ${ }^{1,2}$
}

\footnotetext{
${ }^{1}$ Bethlem Royal Hospital, South London and Maudsley NHS Foundation Trust, London, England

${ }^{2}$ Department of Psychological Medicine, Institute of Psychiatry, Psychology, and Neuroscience, King's College London, London, England
}

Received 25 April 2017; Accepted 24 July 2017; First published online 5 September 2017

Key words: Anorexia nervosa, randomized controlled trials, psychopharmacological treatment.

Dear Editor,

With particular interest, we read the article "Psychopharmacological Options for Adult Patients with Anorexia Nervosa" by Mario Miniati et al. ${ }^{1}$ in CNS Spectrums, in which the evidence from research on psychopharmacological options for adult patients with anorexia nervosa (AN) is summarized. We agree with the authors' view that evidence for the efficacy of pharmacotherapy in $\mathrm{AN}$ is limited due to shortcomings in study design for the majority of published papers, as described in their article, including the lack of a control group or treatment-specific biases arising from small sample sizes. Therefore, there is a need to identify the challenges and barriers to performing high-quality psychopharmacological studies in patients with AN and to discuss how these hurdles can be overcome.

The review by Miniati et al. ${ }^{1}$ discusses the fact that randomized controlled trials (RCTs) often fail, as a considerable number of patients with AN refuse to participate in an RCT or drop out prematurely. In one of the RCTs they cite, by Bissada et al., ${ }^{2}$ it appears that only $19 \%$ of all the patients approached completed the RCT in full, including examination for eligibility, informed consent for participation, randomization, and adherence to treatment for the duration of the study. Therefore, despite this being a double-blind and placebocontrolled study, it may not be representative. One of the problems facing psychopharmacological research in AN could be the reservations patients have regarding drug

* Address for correspondence: Hubertus Himmerich, Department of Psychological Medicine, King's College London, 103 Denmark Hill, London SE5 8AF, United Kingdom.

(Email: hubertus.himmerich@kcl.ac.uk) treatment in general, particularly those that may increase their weight.

In the context of a quality improvement (QI) project on a specialist unit for eating disorders at the South London and Maudsley National Health Service (NHS) Foundation Trust (approved by the Trust's QI team), we surveyed 17 patients with AN and 16 carers. More than half of the patients disagreed $(47 \%)$ or strongly disagreed $(6 \%)$ with the view that medication would be able to reduce AN symptoms, whereas only $6 \%$ of carers disagreed with this statement (and none strongly disagreed). In terms of medication, the views of $41 \%$ of patients and $56 \%$ of carers were neutral, whereas over half of the patients said they would find medication useful if it helped reduce anxiety (53\%) or sleep problems (53\%). In addition, $75 \%$ of patients and $93 \%$ of carers were positive about more scientific research on pharmacological treatment in AN. In $83 \%$ of patients, weight gain as a possible side effect of drug treatment for AN was a concern.

These results indicate that patients with $\mathrm{AN}$, despite believing that medication does not help to treat the symptoms of $\mathrm{AN}$, as well as their carers would, in principle, welcome research on psychopharmacological treatment. Furthermore, the results revealed that the majority of patients with AN would consider medication to treat their anxiety or sleep problems. Considering these data, it is clear that the high proportion of patients with $\mathrm{AN}$ and their carers who are currently neutral regarding medication should be informed and educated about the possible benefits of medication and the rationale behind psychopharmacological treatment for AN to enable them to form their own opinions.

The primary outcome criterion in the majority of RCTs in AN is an increase in body mass index (BMI), ${ }^{1,3}$ as 
the risks of low body weight in $\mathrm{AN}$ are clear from a medical point of view. However, patients might feel that such a trial and such psychopharmacological treatment are just a way to speed up weight gain for the clinician's benefit, rather than to help their own state of mind and anxiety, and that numbers and weight are the only things important for those "higher up."

Therefore, and as patients fear weight gain as a side effect of medication, as indicated by the results of our QI project, the inclusion of patient-reported outcome measures (PROMs) and patient-reported experience measures (PREMs) into the outcome criteria for RCTs should be considered. PROMs and PREMs assess the effectiveness, safety, and experience of care from a patient's perspective. They have already been developed within the NHS of the United Kingdom (UK) for certain elective surgery procedures, ${ }^{4}$ but not yet for the psychopharmacological treatment of AN. If the main outcome criteria of an RCT were to include PROMs and PREMs regarding anxiety and sleep problems in addition to BMI, the RCT would be of benefit from a patient's perspective and might improve their willingness to participate in the trial.

In their article, Miniati et al. ${ }^{1}$ conclude that more high-quality trials are needed while acknowledging that nonresponse and nonremission are typical of patients with AN. In agreement with this statement and in order to achieve RCTs of higher value, we would like to propose a systematic scientific exploration of patients' goals and closer cooperation with patients, their carers, and responsible clinicians with regard to the study design and primary outcome of RCTs. This will assist in meeting the medical requirements and needs of patients in terms of their psychological problems and treatment goals. qIn this respect, we would like to mention the James Lind Alliance. In its priority-setting partnerships (PSPs), the patients, carers, and clinicians unite to prioritize the uncertainties surrounding medical treatment using the UK Database of Uncertainties about the Effects of Treatments (UKDUETs), in which scientific questions and uncertainties regarding treatment are gathered. These PSPs work together to find agreement about which of the questions and uncertainties are most important and to prioritize accordingly. ${ }^{5}$

It remains to be elucidated whether the systematic involvement of patients and carers in research prioritization and study design, as has been begun in the UK with the James Lind Alliance, PSPs, implementation of PREMS and PROMs, use of UKDUETs, and common agreement on research priorities, will improve the quality of RCTs. However, for certain problems, including identifying important outcome measures and overcoming recruitment difficulties, such an approach is likely to be beneficial.

\section{Acknowledgments}

The authors thank Danielle Glennon, Lynn St. Louis, and Mary Cowan for their assistance.

\section{Disclosures}

Hubertus Himmerich, Moradeke Joaquim, Jessica Bentley, Carol Kan, Julia Dornik, and Ulrike Schmidt have no conflicts of interest to disclose.

Janet Treasure reports funding from the BRC Biomedical Research Centre at South London and the Maudsley NHS Foundation Trust and King's College London, the National Institute for Health Research (NIHR), Swiss Anorexia Foundation, Psychiatric Research Trust, and the Guys and St. Thomas Research Trust. She has also received honoraria for participating in the AACAP meeting, Lilly diabetic meeting, ECNP, and Hilda Bruch lecture. She has also received royalties from several books published by Routledge, Wiley, and Oxford University Press.

\section{REFERENCES:}

1. Miniati M, Mauri M, Ciberti A, Mariani MG, Marazziti D, Dell’Osso L. Psychopharmacological options for adult patients with anorexia nervosa. CNS Spectr. 2016; 21(2): 134-142.

2. Bissada H, Tasca GA, Barber AM, Bradwejn J. Olanzapine in the treatment of low body weight and obsessive thinking in women with anorexia nervosa: a randomized, double-blind, placebo-controlled trial. Am J Psychiatry. 2008; 165(10): 1281-1288.

3. Dold M, Aigner M, Klabunde M, Treasure J, Kasper S. Secondgeneration antipsychotic drugs in anorexia nervosa: a meta-analysis of randomized controlled trials. Psychother Psychosom. 2015; 84(2): 110-116.

4. Black N, Varaganum M, Hutchings A. Relationship between patient reported experience (PREMs) and patient reported outcomes (PROMs) in elective surgery. BMJ Qual Saf. 2014; 23(7): 534-542.

5. Petit-Zeman S, Firkins L, Scadding JW. The James Lind Alliance: tackling research mismatches. Lancet. 2010; 376(9742): 667-669. 